\title{
Reductions in caloric intake and early postnatal growth prevent glucose intolerance and obesity associated with low birthweight
}

\author{
J. C. Jimenez-Chillaron • M. Hernandez-Valencia • \\ A. Lightner • R. R. Faucette • C. Reamer • R. Przybyla • \\ S. Ruest • K. Barry • J. P. Otis • M. E. Patti
}

Received: 19 January 2006 / Accepted: 19 April 2006 / Published online: 8 June 2006

(C) Springer-Verlag 2006

\begin{abstract}
Aims/hypothesis Low birthweight (LBW) and rapid postnatal weight gain, or catch-up growth, are independent risk factors for the development of obesity and diabetes during adult life. Individuals who are both small at birth and have postnatal catch-up growth are at the highest risk. We hypothesised that dietary interventions designed to attenuate catch-up growth in LBW subjects may have long-term beneficial consequences.

Materials and methods We used our previously described mouse model of LBW-associated diabetes, created by restricting maternal food intake to $50 \%$ during the last week of gestation. Control (C) dams and dams that had been subjected to undernutrition (U) were then provided either chow ad libitum after delivery or $50 \%$ food restriction on a per-day basis from delivery until weaning. We designated the resulting four groups control-control (CC), undernutrition-control (UC), control-undernutriton (CU) and undernutrition-undernutrition (UU), indicating the prenatal and postnatal experimental conditions, respectively. Carbohydrate metabolism and adiposity were assessed prospectively in offspring until age 6 months.

Results Males that were small at birth and exhibited early postnatal catch-up growth developed glucose intolerance and obesity by age 6 months. In contrast, LBW mice without catch-up growth (UU) remained smaller than controls (CC), and glucose intolerance and obesity was

J. C. Jimenez-Chillaron $\cdot$ M. Hernandez-Valencia $\cdot$ A. Lightner $\cdot$

R. R. Faucette $\cdot$ C. Reamer $\cdot$ R. Przybyla $\cdot$ S. Ruest $\cdot$ K. Barry $\cdot$

J. P. Otis $\cdot$ M. E. Patti $(\bowtie)$

Department of Cellular and Molecular Physiology,

Joslin Diabetes Center,

1 Joslin Place,

Boston, MA 02215, USA

e-mail: mary.elizabeth.patti@joslin.harvard.edu
\end{abstract}

prevented. Similarly, mice with normal birthweight that had blunted catch-up growth (CU) were leaner and had better tolerance test than $\mathrm{CC}$ mice. Catch-up growth during the first week of life correlated better than birthweight with glucose, fat mass and glucose tolerance up to 6 months of age. Conclusions/interpretation Prevention of early catch-up growth reversed the development of glucose intolerance and obesity in our mouse model of LBW-associated diabetes.

Keywords Caloric restriction - Catch-up growth - Diabetes · Growth rate $\cdot$ Intrauterine growth retardation $\cdot$ Low birthweight $\cdot$ Obesity

$\begin{array}{ll}\text { Abbreviations } \\ \text { AGA } & \text { appropriate for gestational age } \\ \text { C } & \text { control } \\ \text { CC } & \text { control-control } \\ \text { CU } & \text { control-undernutriton } \\ \text { DEXA } & \text { dual-energy X-ray absorptiometry } \\ \text { FFM } & \text { fat-free mass } \\ \text { GR } & \text { growth rate } \\ \text { IUGR } & \text { intrauterine growth retardation } \\ \text { LBW } & \text { low birthweight } \\ \text { SGA } & \text { small for gestational age } \\ \text { U } & \text { undernutrition } \\ \text { UC } & \text { undernutrition-control } \\ \text { UU } & \text { undernutrition-undernutrition }\end{array}$

\section{Introduction}

Many epidemiological and animal studies have linked a suboptimal nutritional environment during pregnancy and 
a

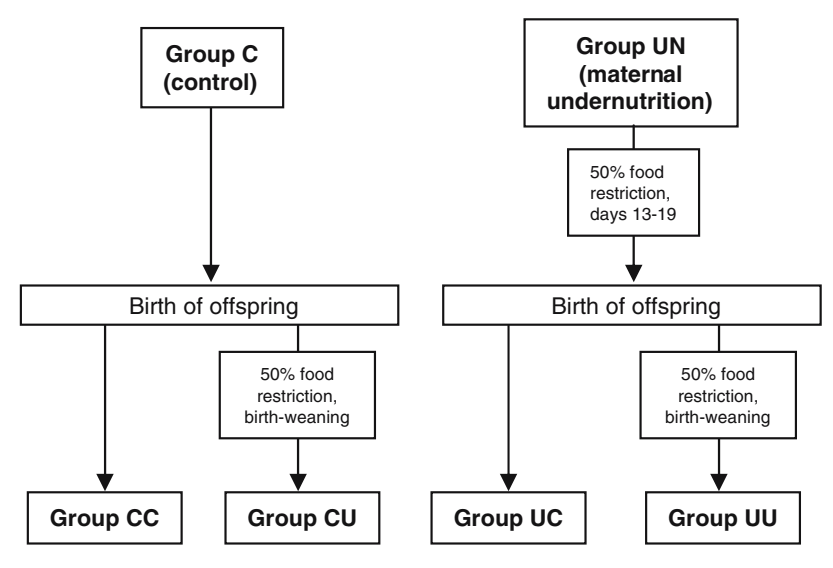

C

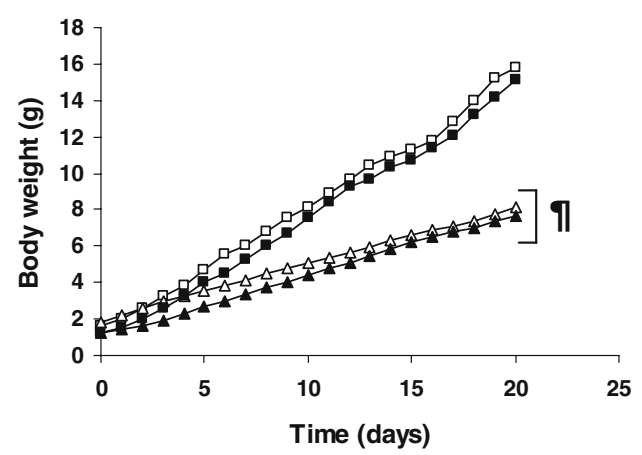

Fig. 1 Experimental design (a), body weight at birth (b), growth curves from birth to day 21 (weaning) (c), and body weight from birth to age 6 months (d). All values (b-d) are mean \pm SEM ( $n \geq 15$ mice/

low birthweight (LBW) with increased susceptibility to chronic disease during adult life [1-3]. The developmental programming hypothesis proposes that insults or stimuli acting during critical windows of development, including fetal and/or early postnatal periods, can produce permanent alterations in cell/tissue structure and function [4].

A growing body of evidence suggests that rapid postnatal weight gain, or catch-up growth, is also associated with increased susceptibility to obesity and type 2 diabetes $[5,6]$. The risk associated with catch-up growth is even higher when catch-up growth operates during periods characterised by rapid increments in weight and length/ height, such as early postnatal life [6-8]. Such patterns of accelerated postnatal growth are common in individuals who are small for gestational age (SGA) and may be considered a physiological adaptation that allows them to reach their genetically programmed weight and/or height after a period of growth retardation $[9,10]$. Not surprisingly, individuals who are both small at birth and have postnatal catch-up growth are at high risk of (central) obesity, glucose intolerance and diabetes [8, 9, 11-14]. b
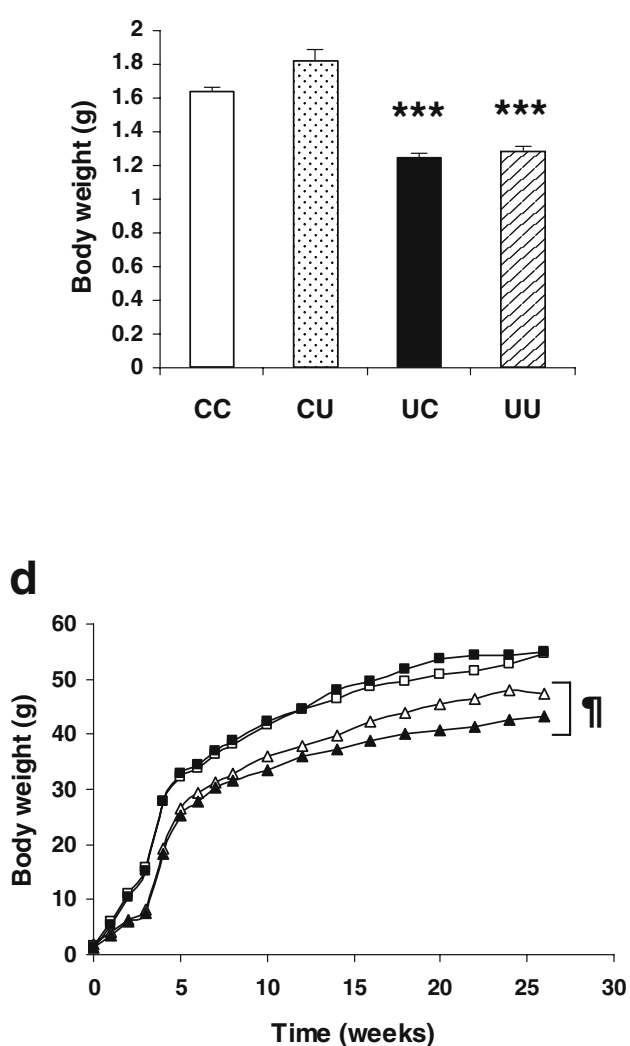

group). Open squares, CC; open triangles, CU; filled squares, UC; filled triangles, UU. $* * * p<0.001$ vs $\mathrm{CC}$ as assessed by Student's $t$-test. $p<0.0001$ vs CC as assessed by repeated measures ANOVA

Thus, rapid postnatal growth in either SGA children or children who are appropriate for gestational age (AGA) may contribute significantly to the development of metabolic syndrome during adult life. Here, we postulate that dietary interventions designed to limit catch-up growth in LBW infants or to reduce early postnatal growth rates in AGA infants may have beneficial long-term consequences. To test this hypothesis, we used our mouse model of LBWassociated diabetes [15]. In this model, maternal nutrient restriction during the final week of gestation reduces birthweight in offspring. Normalisation of maternal nutrition at delivery is associated with catch-up growth in offspring, such that offspring weight normalises by the age of 3 weeks. Since nutrient availability is a key determinant of postnatal growth, we restricted maternal food availability during the 3 weeks of lactation in order to prevent early postnatal catch-up growth in LBW mice. In this context, we evaluated the relative contributions of intrauterine growth retardation (IUGR) and catch-up growth to the development of adult metabolic phenotypes, including body weight, fat mass and glucose homeostasis. Moreover, we defined the 
potential critical period(s) of growth that can influence these adult metabolic phenotypes.

\section{Materials and methods}

Animal care and experimental protocol

Animal protocols were approved by the Joslin Animal Care and Use Committee. All mice were housed in an animal facility approved by the Office of Laboratory Animal Welfare, with controlled temperature, humidity and lightdark cycle. Six- to eight-week-old virgin female ICR mice (Harlan, Indianapolis, IN, USA) were caged with ICR males. Pregnancy was dated with vaginal plugs (day 0.5). Females were subsequently housed individually with free access to Purina 9F (Purina Mills, St Louis, MO, USA) rodent chow $(21 \%$ of calories derived from protein, $21 \%$ from fat, $56 \%$ from carbohydrate). On pregnancy day 12.5 , females were randomly assigned to either a control (C) group or a group subjected to undernutrition (U) (Fig. 1a); there were no differences in weight between $\mathrm{C}$ and $\mathrm{U}$ mothers before pregnancy or at the time of group assignment. Food intake of $U$ mothers was restricted to $50 \%$ of that of controls, calculated on a per gestational day basis, from day 12.5 to 18.5 . After delivery, litter size was equalised to 8 in all groups. $\mathrm{C}$ and $\mathrm{U}$ litters were further distributed into two additional experimental groups: mothers in both control and $U$ groups were provided with either (1) free access to chow after delivery, or (2) $50 \%$ food restriction on a per day basis from the day of delivery until the day of weaning ( 21 days post partum). We designated these four groups as control-control (CC), undernutritioncontrol (UC), control-undernutriton (CU) and undernutrition-undernutrition (UU), indicating the prenatal and postnatal experimental condition, respectively. For all groups, pups were weaned at age 3 weeks onto Purina 9F chow, to which they had free access. Male offspring were followed longitudinally up to age 12 months because of the higher prevalence of glucose intolerance and diabetes in male LBW mice, as previously noted [15].

Growth rate

Body weight was recorded weekly on each mouse from birth to age 6 months. Specific growth rate was calculated as the specific weekly weight gain, corrected by the body weight at the beginning of each week interval:

$$
\mathrm{GR}=\left(\mathrm{W}_{\mathrm{n}}-\mathrm{W}_{\mathrm{n}-1}\right) / \mathrm{W}_{\mathrm{n}-1}
$$

where GR is growth rate, $W$ is body weight, and $n$ is age in weeks.

\section{Dual-energy X-ray absorptiometry scanning}

Body composition was analysed using dual-energy X-ray absorptiometry (DEXA) (Hologic, Waltham, MA, USA), as described [16].

Serum analysis

Insulin and leptin were measured in $5 \mu \mathrm{l}$ serum samples by ELISA (Crystal Chem, Downers Grove, IL, USA). Wholeblood glucose was measured with a Glucometer Elite (Bayer, Elkhart, IN, USA). Total adiponectin was measured by ELISA in $5 \mu \mathrm{l}$ aliquots of serum diluted at 1:100 (Linco Research, St Charles, MO, USA).

Statistical analysis

Results are expressed as mean \pm SEM. For group comparisons, statistical analysis was performed using a two-tailed $t$-test or ANOVA (Statview, Cary, NC, USA); $p$ values $\leq$ 0.05 were considered significant.

\section{Results}

Growth effects of early postnatal reductions in caloric intake

As previously reported [15], 50\% global caloric restriction during the last week of gestation produced a $25 \%$ decrease in body weight at birth ( $\mathrm{UC}$ vs CC mice, $p<0.001$ ) (Fig. 1b). Length of pregnancy was similar in all groups (data not shown), and pup number was slightly, although not significantly, reduced in the pregnancies with maternal undernutrition (CC, $10 \pm 1.0$ mice/litter; $\mathrm{CU}, 11 \pm 1.6$; UC, $8.5 \pm 0.5$; UU, $8.2 \pm 0.9$ ). Litters were normalised to eight pups in all groups. As previously demonstrated, UC mice exhibited catch-up growth, with weights similar to $\mathrm{CC}$ offspring by the third week of life (Fig. 1c). In contrast, offspring in postnatal food restriction groups (CU, UU) showed a $50 \%$ reduction in body weight at the time of weaning compared with CC (Fig. 1c). Interestingly, UU mice, which were smaller at birth $(25 \%$ decrease relative to $\mathrm{CC}$ ), also showed catch-up growth (relative to $\mathrm{CU}$ mice), reaching the same body weight as $\mathrm{CU}$ mice by 3 weeks of life. While CC and UC mice were similar in weight up to age 6 months, weights of CU and UU mice remained significantly lower than those of $\mathrm{CC}$ mice despite ad libitum feeding after weaning (Fig. 1d).

While it is difficult to measure food intake accurately in offspring mice during the suckling period, we carefully assessed food intake at 2 and 4 months of age (Table 1). As shown, food intake was similar in CC and UC mice at these 
Table 1 Food intake at 2 and 6 months of age

\begin{tabular}{llllll}
\hline Group & 2 months & & & \multicolumn{2}{l}{6 months } \\
\cline { 2 - 3 } \cline { 6 - 7 } & Food (g/day) & Food/BW & & Food (g/day) & Food/BW \\
\hline CC & $3.76 \pm 0.48$ & $0.1 \pm 0.01$ & & $3.36 \pm 0.24$ & $0.06 \pm 0.005$ \\
CU & $4.45 \pm 0.18$ & $0.15 \pm 0.007^{\mathrm{a}}$ & & $3.48 \pm 0.25$ & $0.08 \pm 0.005$ \\
UC & $3.45 \pm 0.47$ & $0.09 \pm 0.01$ & & $3.72 \pm 0.83$ & $0.08 \pm 0.02$ \\
UU & $3.31 \pm 0.37$ & $0.12 \pm 0.01$ & & $3.08 \pm 0.36$ & $0.07 \pm 0.006$ \\
\hline
\end{tabular}

Data are mean \pm SEM

${ }^{\mathrm{a}} p<0.05$ vs $\mathrm{CC}$

$B W$ Body weight

ages. Interestingly, food intake (corrected for body weight) was increased in $\mathrm{CU}$ mice, despite their remaining smaller and leaner than $\mathrm{CC}$ mice.
We next modelled the specific growth rate in our mouse cohorts as described in Materials and methods. Two distinct growth spurts were observed in control mice, the first occurring during the first week of life and the second after weaning, between the 3rd and 5th weeks (Fig. 2a). After 7 weeks, growth rate remained constant, and was similar in all groups until age 6 months. UC mice, which exhibited catch-up growth, had a significantly higher specific growth rate than $\mathrm{CC}$ mice during the first and second weeks of life (Fig. 2b). By contrast, growth rates of CU and UU mice (which were food-restricted during the suckling period) were reduced during early postnatal life (Fig. 2b) compared with $\mathrm{CC}$ and UC offspring. As predicted, growth rates in CU and UU cohorts increased between 4 and 6 weeks of life, following the onset of ad libitum feeding at weaning (Fig. 2b).
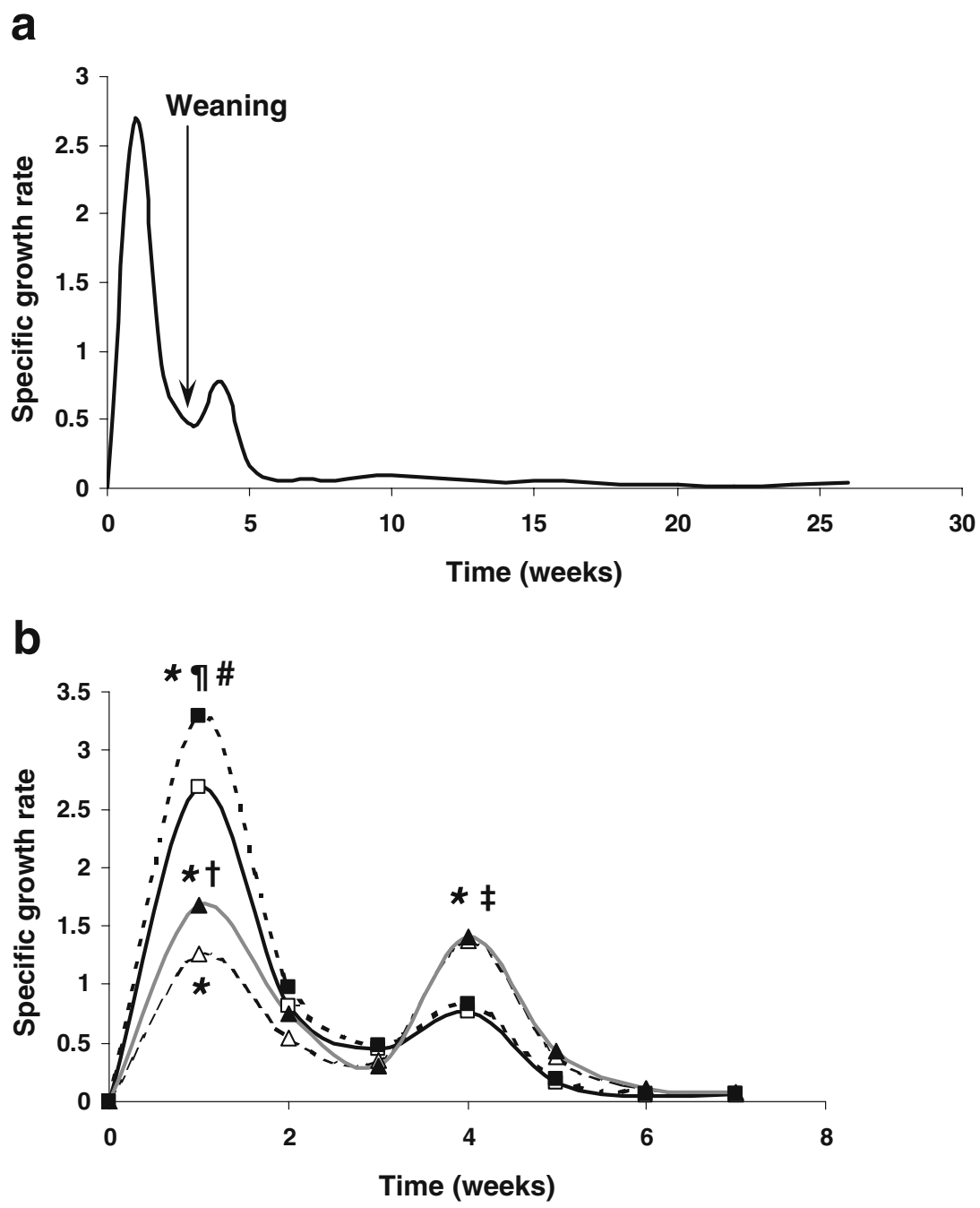

Fig. 2 Specific growth rate patterns are altered by prenatal and postnatal nutritional intervention. a Specific growth rate profile of CC mice from birth to age 26 weeks, calculated as described in Materials and methods. b Specific growth rate of the four experimental groups from birth to age 7 weeks. Open squares, $\mathrm{CC}$; open triangles, $\mathrm{CU}$; filled squares, UC; filled triangles, UU. Values are means $(n \geq 15$ animals/group). * $p<0.0001$ vs CC, $\mid p<0.0001$ vs CU, \# $p<0.0001$ vs $\mathrm{UU}, \dagger p<0.005$ vs UU, $\ddagger p<0.0001$ vs UC ( $t$-test) 
Fig. 3 Glucose and insulin tolerance in 6-month-old male mice. a Random fed glucose levels and $\mathbf{b}$ glucose levels during an intraperitoneal glucose tolerance test ( $2 \mathrm{~g}$ glucose/ $\mathrm{kg}$ body weight), performed after an overnight fast. ${ }^{*} p<0.05$ vs CC (repeated measures ANOVA). c Glucose AUC during the glucose tolerance test. $* p<0.05$ vs UC, $* * p<0.01$ vs UC. d Glucose measurements during intraperitoneal insulin tolerance test $(1 \mathrm{U} / \mathrm{kg}$ body weight), performed after a 4-h fast. Results are expressed as mean \pm SEM ( $n \geq 6$ mice/ group). Open squares, CC; open triangles, CU; filled squares, UC; filled triangles, UU

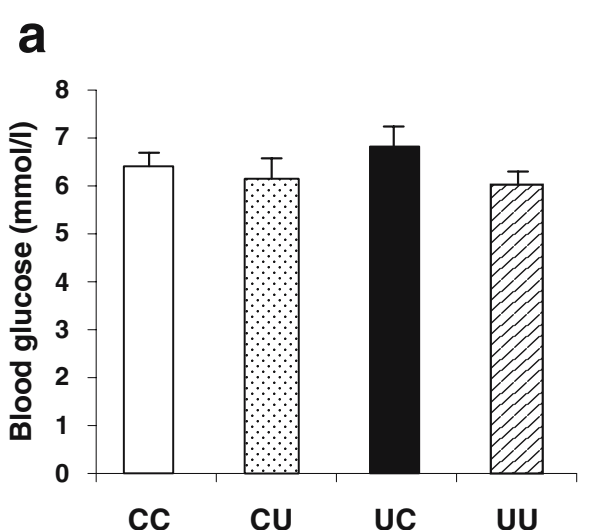

b
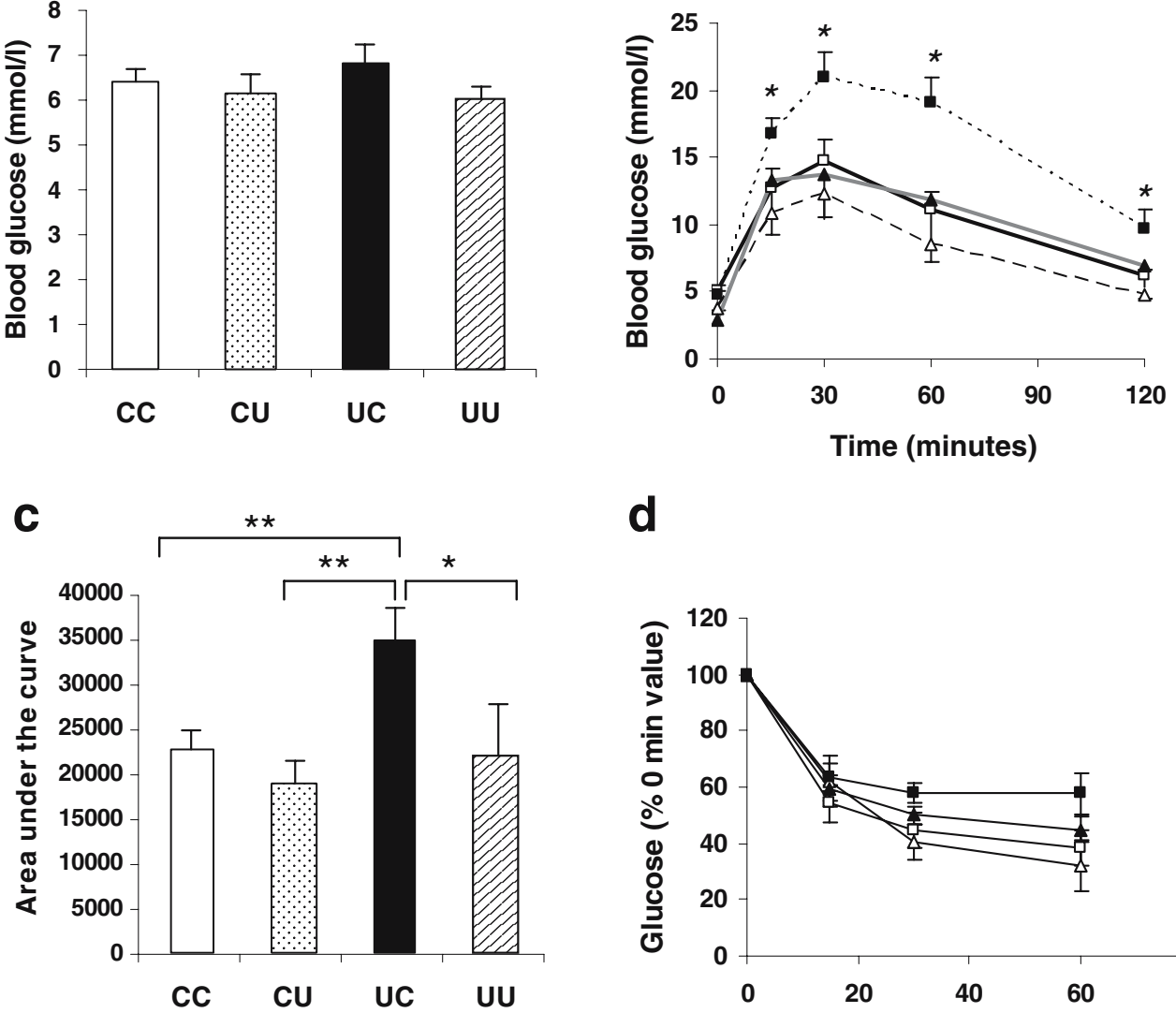

d

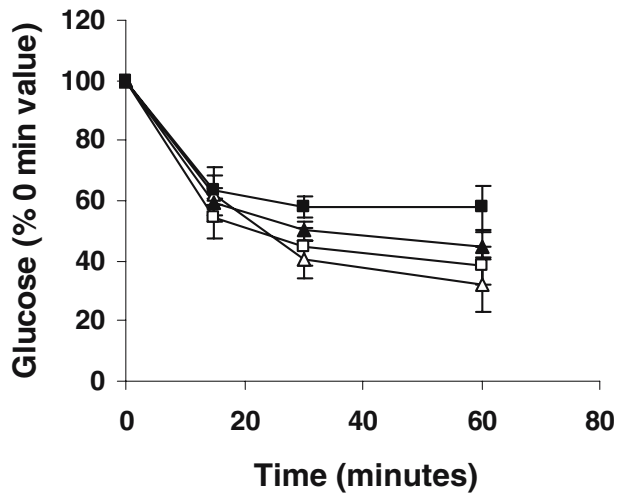

Animal physiology

We have previously shown that LBW mice develop progressive glucose intolerance during adult life [15]. As expected, UC mice maintained glucose homeostasis in the fed and fasted states (Fig. 3a) but developed glucose intolerance by age 6 months (Fig. 3b). By contrast, LBW mice with continued postnatal dietary restriction (UU) were completely protected from the development of glucose intolerance during adult life, despite being small at birth. It is also interesting that both $\mathrm{CU}$ and UU mice had normal glucose levels and normal glucose tolerance (Fig. 3a-c), irrespective of their weight at birth and despite some accelerated growth occurring after age 3 weeks. There were no differences in insulin sensitivity among the four groups, as assessed by intraperitoneal insulin tolerance testing at age 6 months (Fig. 3d).

To address potential hormonal factors responsible for between-group differences in age-dependent glucose tolerance, we measured insulin in the fed state at ages 2 and 6 months. Fed insulin levels were 35 to $50 \%$ lower in $\mathrm{CU}$ and UU mice than in CC mice $(p<0.05$ for both comparisons at 2 months) (Fig. 4a,b). Since insulin sensitivity was similar in all groups, these data suggest that the abnormalities in insulin secretion and/or clearance previously observed in U mice [15] can be modulated by postnatal dietary intervention.

Catch-up growth is mediated in part by accelerated fat mass accretion [7]. Therefore we measured total body fat content by DEXA (Table 2). At both 6 and 12 months of age, body fat mass, expressed as a percentage of body weight, was significantly higher in LBW mice with catchup growth (UC) than in CC mice. However, early postnatal caloric restriction in UU mice prevented the obesity seen in LBW mice with catch-up growth. Interestingly, while body weight was lower in both CU and UU mice as compared with CC mice, their relative fat content (percentage of body weight) was similar to that of CC offspring. Similarly, abdominal fat mass was disproportionately large in UC mice. These patterns were even more striking at 12 months of age.

We also assessed fat-free mass (FFM) in offspring mice (Table 2). Total FFM was similar between CC and UC mice, whereas it was significantly reduced in $\mathrm{CU}$ and $\mathrm{UU}$ mice at 6 months and in UU mice at 12 months. Since CU and UU mice have reduced body weight, we also assessed 
Fig. 4 Insulin at 2 (a) and 6 (b) months, as well as leptin (c) and adiponectin (d) were measured in serum samples from 6-month-old male mice in the fed state. Results are expressed as mean \pm SEM $(n=15$ mice/group). $* p<0.05$ for $\mathrm{CC}$ vs $\mathrm{CU}$ and $\mathrm{CC}$ vs UU

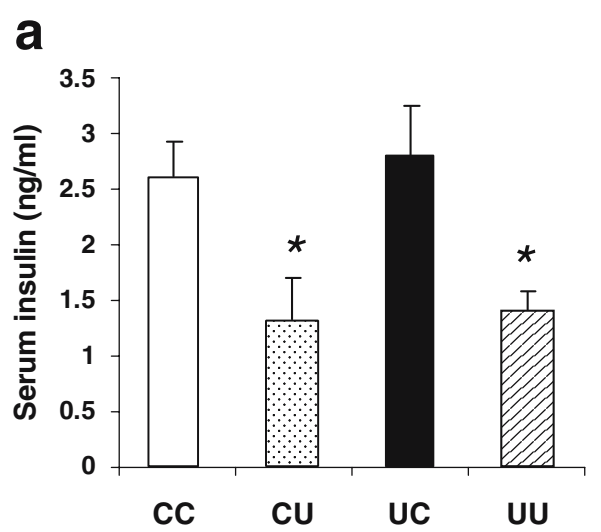

b
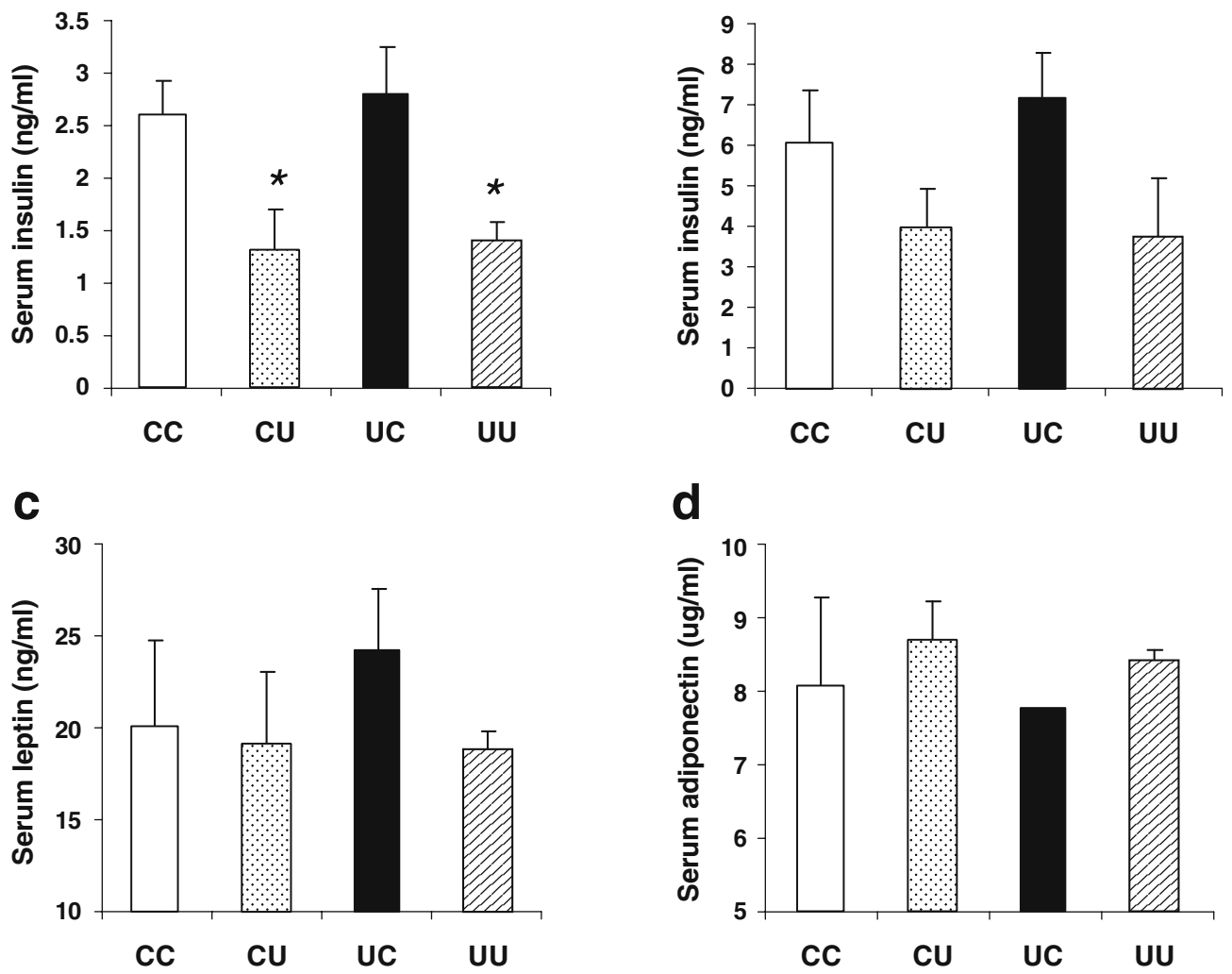

d

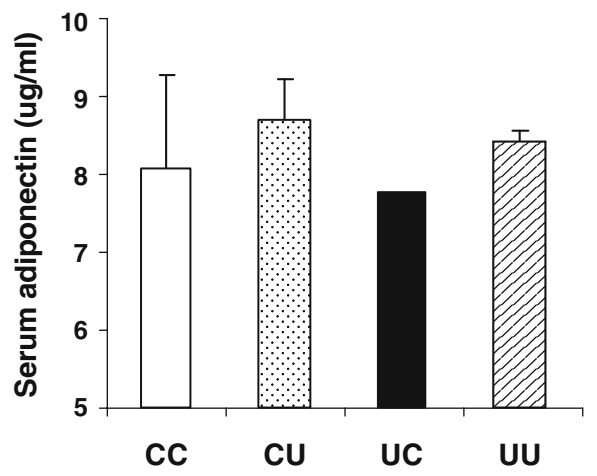

relative FFM; relative FFM was preserved in CU and UU mice. Thus, the overall ratio of fat mass to FFM (28 vs $72 \%$ ) was increased in UC mice but remained normal in UU mice (20 vs 80\%).

We next assessed serum markers of adipocyte mass and function. Serum leptin levels paralleled fat mass in all groups ( $r=0.67, p=0.001$ ), with a trend to higher levels in LBW mice with catch-up growth (UC) (Fig. 4c). Levels of adiponectin, another fat-derived adipokine, tended to be lower in UC offspring than in CU or UU mice by 6 months of age (Fig. 4d).
Correlation analysis

In order to estimate the individual contributions of birthweight and postnatal growth to the development of obesity and glucose intolerance, we performed regression analysis across all experimental groups. As shown in Table 3, birthweight did not correlate significantly with body weight, glucose, insulin, fat mass or glucose homeostasis (AUC) at 6 months of age. We next analysed the correlation between growth rates (GR) during the first 6 weeks of life (GR 1-6) and metabolic phenotypes, since this is the period of catch-up growth during which growth rate differed between experimental groups. By contrast to the lack of

Table 2 Body weight, fat mass and fat-free mass of 6- and 12-month-old male mice

\begin{tabular}{|c|c|c|c|c|c|c|c|c|}
\hline & \multicolumn{4}{|l|}{6 months } & \multicolumn{4}{|c|}{12 months } \\
\hline & $\mathrm{CC}$ & $\mathrm{CU}$ & UC & UU & $\mathrm{CC}$ & $\mathrm{CU}$ & $\mathrm{UC}$ & UU \\
\hline Body weight (g) & $52.8 \pm 2.0$ & $45.6 \pm 2.3^{\mathrm{a}}$ & $54.9 \pm 3.0$ & $41.4 \pm 3.0^{\mathrm{b}}$ & $54.6 \pm .5$ & $47.9 \pm 2.7$ & $61.9 \pm 2.5^{\mathrm{a}}$ & $45.1 \pm 3.8^{\mathrm{a}}$ \\
\hline Fat mass $(\mathrm{g})$ & $12.3 \pm 1.0$ & $10.5 \pm 1.2$ & $15.1 \pm 2.1$ & $8.6 \pm 1.5$ & $15.5 \pm 2.4$ & $10.4 \pm 1.7$ & $21.5 \pm 1.8 \mathrm{a}$ & $12.7 \pm 2.5$ \\
\hline Relative body fat (\%) & $22.7 \pm 1.3$ & $22.7 \pm 1.8$ & $27.5 \pm 1.8^{\mathrm{a}}$ & $20.2 \pm 2.4$ & $20.7 \pm 0.3$ & $16.7 \pm 2.7$ & $29.1 \pm 1.3^{\mathrm{a}}$ & $23.2 \pm 3.2$ \\
\hline Abdominal fat (\%) & $6.9 \pm 0.6$ & $6.2 \pm 0.7$ & $7.7 \pm 1.0$ & $5.5 \pm 0.9$ & $5.3 \pm 1.0$ & $4.0 \pm 1.0$ & $8.7 \pm 0.6^{\mathrm{b}}$ & $6.4 \pm 1.1$ \\
\hline Fat-free mass (g) & $39.7 \pm 1.3$ & $35.1 \pm 1.6^{\mathrm{a}}$ & $39.8 \pm 1.1$ & $32.7 \pm 1.7^{\mathrm{a}}$ & $39.1 \pm 0.4$ & $37.6 \pm 1.5$ & $40.4 \pm 1.2$ & $32.4 \pm 1.8^{\mathrm{a}}$ \\
\hline Relative fat-free mass (\%) & $76.4 \pm 1.3$ & $77.2 \pm 1.9$ & $72.5 \pm 2.5$ & $79.9 \pm 2.3$ & $72.2 \pm 3.2$ & $78.7 \pm 2.5$ & $65.4 \pm 0.9$ & $72.7 \pm 3.4$ \\
\hline
\end{tabular}

Data are mean \pm SEM

${ }^{\mathrm{a}} p<0.05,{ }^{\mathrm{b}} p<0.01$ vs CC 
Table 3 Correlations of birthweight (BW) and growth rate (GR) with adult phenotypes at 6 months

\begin{tabular}{|c|c|c|c|c|c|c|c|c|c|c|c|c|}
\hline & \multicolumn{2}{|c|}{ Birthweight } & \multicolumn{2}{|c|}{$\begin{array}{l}\text { GR }(1- \\
6 \text { weeks })\end{array}$} & \multicolumn{2}{|c|}{ GR (1st week) } & \multicolumn{2}{|c|}{ GR (4th week) } & \multicolumn{2}{|c|}{ GR (1-3 weeks) } & \multicolumn{2}{|c|}{ GR (4-6 weeks) } \\
\hline & $r$ & $p$ & $r$ & $p$ & $r$ & $p$ & $r$ & $p$ & $r$ & $p$ & $r$ & $p$ \\
\hline Glucose & 0.06 & 0.60 & 0.05 & 0.68 & 0.01 & 0.91 & 0.09 & 0.43 & 0.03 & 0.80 & 0.06 & 0.64 \\
\hline Insulin & 0.08 & 0.62 & 0.34 & 0.04 & 0.23 & 0.13 & 0.24 & 0.13 & 0.35 & 0.02 & -0.30 & 0.07 \\
\hline Leptin & -0.04 & 0.73 & 0.15 & 0.27 & 0.07 & 0.57 & 0.08 & 0.53 & 0.13 & 0.32 & -0.12 & 0.38 \\
\hline Adiponectin & 0.08 & 0.48 & 0.04 & 0.75 & 0.04 & 0.73 & 0.03 & 0.81 & 0.09 & 0.46 & 0.008 & 0.95 \\
\hline \multicolumn{13}{|l|}{ Body weight } \\
\hline 6 months & 0.18 & 0.12 & 0.28 & 0.02 & 0.46 & $<0.0001$ & -0.58 & $<0.0001$ & 0.56 & $<0.0001$ & -0.61 & $<0.0001$ \\
\hline 12 months & 0.09 & 0.42 & 0.33 & 0.01 & 0.41 & 0.006 & -0.38 & 0.001 & 0.47 & 0.0001 & -0.43 & 0.0008 \\
\hline \multicolumn{13}{|l|}{ Total body fat } \\
\hline 6 months & 0.01 & 0.91 & 0.04 & 0.84 & 0.29 & 0.08 & -0.18 & 0.26 & 0.23 & 0.25 & -0.28 & 0.17 \\
\hline 12 months & 0.18 & 0.41 & 0.60 & 0.003 & 0.54 & 0.005 & -0.30 & 0.14 & 0.59 & 0.003 & -0.40 & 0.07 \\
\hline \multicolumn{13}{|l|}{ Abdominal fat } \\
\hline 6 months & 0.04 & 0.81 & 0.16 & 0.42 & 0.27 & 0.16 & -0.22 & 0.27 & 0.31 & 0.12 & -0.34 & 0.10 \\
\hline 12 months & 0.27 & 0.21 & 0.68 & 0.0007 & 0.57 & 0.004 & -0.28 & 0.17 & 0.64 & 0.001 & -0.42 & 0.05 \\
\hline $\begin{array}{l}\text { GTT AUC at } \\
6 \text { months }\end{array}$ & 0.28 & 0.10 & 0.62 & 0.0006 & 0.43 & 0.006 & -0.21 & 0.21 & 0.41 & 0.01 & -0.43 & 0.02 \\
\hline
\end{tabular}

Data are mean \pm SEM

GTT Glucose tolerance test

correlation with birthweight, GR 1-6 was significantly positively correlated with body weight, fat mass (whole body and abdominal), insulin, and glucose tolerance (AUC).

We next wished to determine which time period(s) within the first 6 weeks of life accounted for the correlations between early postnatal growth and adult metabolic phenotypes. We focused on growth rates during the first 3 weeks (GR 1-3) and the fourth to sixth (GR 4-6) weeks of life, since these are the two periods that coincided with growth spurts and during which differences between groups were most marked. GR 1-3 strongly correlated with body weight, fat mass (whole-body and abdominal), insulin and glucose AUC (Table 3). GR 4-6 correlated only with body weight and abdominal fat. Within the first 3 weeks of life, the growth rate during week 1 was most strongly linked to metabolic phenotypes. Indeed, the regression coefficients were similar in magnitude and direction to those for GR 1-6, suggesting that growth rate during the first week of life is a major contributor to the final adult phenotype. Growth rates during week 4 of life, in contrast, were inversely correlated with body weight. Taken together, these data suggest that catch-up growth during the first week of life is a dominant factor in the development of adult metabolic disturbances in our mouse model of LBW.

\section{Discussion}

Our data demonstrate that prevention of early catch-up growth prevents the development of glucose intolerance and obesity in our mouse model of LBW-associated diabetes. Two important implications arise from this finding: (1) rapid early postnatal growth is more detrimental than LBW per se for the development of adult metabolic phenotypes, at least in our experimental model; and (2) in agreement with the developmental programming hypothesis [17], both fetal and early postnatal periods are critical windows during which perturbations in nutritional status can have long-term consequences. Furthermore, these data suggest that prenatal metabolic adaptations are not completely fixed and may be reversed by subsequent postnatal interventions.

Extensive epidemiological data indicate that both LBW and catch-up growth are associated with higher susceptibility to adult chronic disease [13]. In most of these studies, catch-up growth appears in association with intrauterine growth restriction [8, 9, 11, 12, 15-19], making it difficult to interpret the relative contribution of each variable (IUGR with LBW, vs catch-up growth) to disease risk. In this study, we dissociated catch-up growth from LBW by experimental nutritional interventions, thus assessing the independent contributions of these factors to adult metabolic phenotypes. We now show that LBW mice that exhibit early postnatal catch-up growth (UC mice) develop obesity and glucose intolerance. In contrast, LBW mice that do not exhibit catch-up growth relative to CC mice (UU mice) are protected from developing obesity and glucose intolerance, at least on a chow diet. Furthermore, mice with birthweights within the normal range but with blunted early postnatal growth (CU mice) have reduced body weight and better glucose tolerance than control (CC) mice, suggesting 
Fig. 5 Schematic relationships between growth rate and adult phenotype. Growth rates of experimental groups are presented relative to control mice. Thus, relative growth retardation is represented by negative values, while accelerated growth is represented by positive values

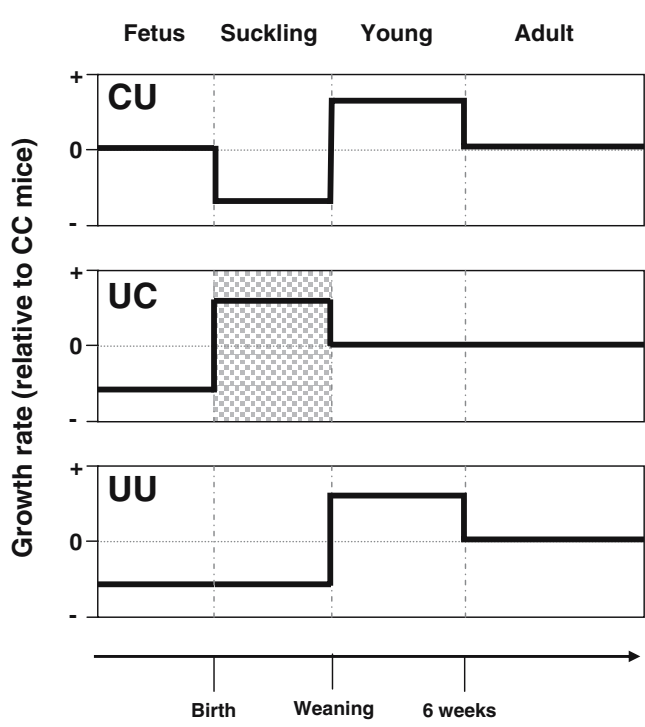

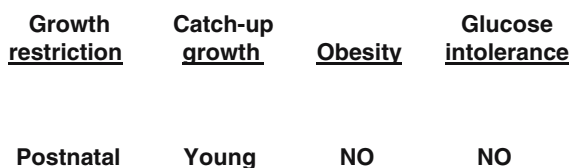

Prenatal Postnatal YES YES

$\begin{gathered}\text { Prenatal Young NO NO } \\ \text { and }\end{gathered}$
Pos

that reduced postnatal growth could be protective against adult disease. Together, these data imply that, at least in our mouse model, the combination of LBW and early postnatal catch-up growth potentiates the risk of adult phenotypes (Fig. 5). In contrast, catch-up growth occurring during the post-weaning period (young adult life) does not contribute to the development of obesity or glucose intolerance, irrespective of body weight at birth. Thus, our data suggest that it may be possible to design postnatal interventions aimed at attenuating early catch-up growth and ultimately reducing the risk of adult diseases.

Several recent studies have begun to address the effects of postnatal nutritional and hormonal interventions on later disease risk in rodents. For example, female offspring of rats maintained on a low-protein diet $(8 \%)$ throughout pregnancy and lactation were protected from later obesity but developed hypertension compared with controls [18]. In addition, normal-weight mice that were nursed by dams fed a low-protein diet showed slow postnatal growth rate, reduced body weight and increased longevity compared with controls $[19,20]$. Moreover, postnatal dietary supplementation with $\omega-3$ fatty acids can prevent hyperleptinaemia and hypertension in offspring of mothers treated with dexamethasone during pregnancy [21]. Conversely, postnatal overfeeding in rats to accelerate postnatal growth reproduces some aspects of the human phenotype, including increased risk of later development of obesity, hyperinsulinaemia, hypercholesterolaemia and diabetes [22]. Finally, two recent studies have indicated a role for alterations in the early neonatal secretion and/or action of leptin, a key hormonal sensor and effector of nutrient adequacy, in modulating LBW-associated metabolic phenotypes. Neonatal leptin surges occurred earlier in $U$ offspring than in controls [23], while pharmacological doses of leptin administered to LBW rat pups during the nursing period prevented obesity and hyperleptinaemia with high-fat feeding [24]. While discordant in some aspects, these papers suggest an important role for the timing and magnitude of neonatal leptin in influencing the risk of obesity in later life [25]. Together with our data, these studies indicate that manipulation of postnatal nutrition and hormonal responses can prevent developmental programming. Nevertheless, it remains unclear whether the nutritional and hormonal interventions that have been described have an impact on early growth rate, and whether the reported effects on adult phenotypes are mediated through the modulation of postnatal catch-up growth.

In our experiment, modulation of postnatal catch-up growth was achieved through relative calorie restriction during the suckling period. Similar findings have been reported in humans, where preterm LBW babies that were fed a low-nutrient diet during the first 2 weeks of life had reduced postnatal growth rates but lower markers of insulin resistance and improved endothelial function by age 13 to 16 years $[14,26]$. These authors concluded that relative undernutrition early in life ( 2 weeks after birth) may have long-term beneficial effects. Conversely, overnutrition of AGA children in terms of either high-protein [27] or rich milk formulas [28] has been linked to increased C-peptide levels and high blood pressure, respectively. Animal and human data, considered together, suggest that postnatal nutritional interventions can have potent effects on the later risk of chronic disease.

Still, we recognise that postnatal undernutrition may not be a viable strategy to modulate growth rate in SGA and/or AGA children because of concerns that inadequate nutrition 
during early postnatal life could have adverse effects on central nervous system development, cognitive function and the risk of infection [29-31]. An important challenge will be to find strategies that balance adequate nutritional status and postnatal growth while preventing excess catchup growth. Future investigation will be focused on components and/or markers of the metabolic and hormonal milieu that may mediate postnatal catch-up growth, including maternal milk composition as influenced by diet.

An important question that we hoped to elucidate concerns the developmental window(s) during which (accelerated) postnatal weight gain has a critical effect on adult health. To this end, we profiled the specific growth rate in our mouse cohorts from birth to 6 month of age. As described, growth rate was highest during the first 6 (GR 1-6) weeks of life, remaining at very low and constant levels beyond this age. Growth rate during this early stage of life was characterised by two growth spurts occurring during the first and fourth weeks of life, periods that could be considered equivalent to infancy and adolescence in humans [6]. Here we show that growth rate during the first week of life (GR 1) has the strongest positive correlation with body weight, fat mass, insulin and glucose tolerance by 6 months of age, implying that the faster the growth during this period, the higher the risk of obesity and glucose intolerance. This suggests that the first week of life appears to be the most critical period of development that can influence adult health in the mouse. Furthermore, these data imply that modulation of growth during this time frame may have long-term consequences for health outcomes. Additional experiments, with daily assessment of growth rates, will be required to narrow the critical period(s) that contribute to increased risk of disease in our mouse cohorts. This will allow us to develop postnatal interventions intended to restrict growth rate only during this period(s) of maximal susceptibility to developmental programming.

In humans, there is some conflict regarding the critical period(s) of growth that influence(s) adult phenotype. Nevertheless, the general consensus is that, in agreement with our data, early infancy is the most critical window, since catch-up postnatal growth is largely seen within the first 2 years [32]. Some authors report that the two first weeks of life $[14,26,33]$ can have long-term consequences, while others suggest windows within $1-3$ years $[5,34-36]$ or 4-11 years [37-39]. The variability of these conclusions may be related to study design, since in some cases data from early stages of life (first weeks or months) were not recorded, with the first postnatal data collection only several years after birth. This makes it difficult to profile accurately the very early stages of life and analyse their impact on adult outcomes. More work needs to be done to profile early periods of life in humans and to determine their potential influence on health outcomes.
Increased adiposity is a serious complication of LBW and subsequent catch-up growth in rodents and humans [36]. While our initial cohort of $U$ mice did not have significantly increased fat mass, as determined by carcass analysis [15], we detected significantly increased fat in both whole-body and abdominal compartments by DEXA analysis in two independent cohorts. Although we cannot completely address the reason(s) that could account for this difference, we speculate that subtle differences in fat mass were not detected by the original methods. Development of obesity in UC mice suggests that catch-up growth actually reflects fat mass accretion (i.e. catch-up fat). Conversely, fat mass is reduced in UU mice that are unable to catch up in weight to matched controls. It is also important to note that relative FFM, which plays a central (positive) role in glucose homeostasis, is preserved in UU and CU mice but slightly reduced in UC mice. Thus, dysregulated glucose homeostasis in UC mice may be related not only to increased fat accretion, but also to an overall shift in body composition (as reflected by the increased fat/FFM ratio).

What are the mechanisms underlying increased adiposity in relationship to catch-up growth? In rats, catch-up growth after a period of semi-starvation is characterised by accelerated and preferential recovery of fat mass compared with lean mass $[9,40]$, an effect potentially mediated by suppressed muscle thermogenesis, resulting in a shift of nutrients (glucose) to adipose tissue [40]. To find out whether a similar mechanism accounts for the increased fat accretion in UC mice during early stages of growth will require additional studies. Several other mechanisms may contribute to increased adiposity in relationship to catch-up growth. For example, increased food intake may contribute to increased adiposity in UC mice. While we do not have any measurement of food intake during the suckling period, when catchup growth occurs food intake is similar in UC mice compared with $\mathrm{CC}$ mice at both 2 and 6 months of age. While these data indicate that postweaning food intake does not account for increased adiposity in UC mice, additional experiments will be required to evaluate potential differences in energy intake during the suckling period. We speculate that dysregulation of nutrient-stimulated insulin secretion, favouring inappropriate degrees of hyperinsulinaemia, may also contribute to fat accretion in our model. Other hormones and growth factors may also alter body composition in the setting of catch-up growth, including insulin-like growth factors, glucocorticoids or leptin [41, 42], or altered expression of genes regulating fat tissue development and/or differentiation [10]. Finally, altered timing and patterns of critical hormonal responses, including leptin, may contribute to the disruption of central nervous system architecture and function. This may lead, in turn, to programmed changes in leptin sensitivity and the balance of other pathways regulating satiety and energy expenditure - an important area for future investigation $[23,25]$. 
In conclusion, our data demonstrate that rapid postnatal growth, independent of birthweight, is a major contributor to later risk of adult disease. Preventing postnatal catch-up growth by modulating nutrient availability in LBW mice completely reverses the programming of obesity and glucose intolerance. In addition, deceleration of early growth rate in normal weight mice also improves glucose tolerance by age 6 months. These data suggest that reducing early postnatal growth rate in both SGA and AGA children could have potential beneficial consequences for long-term health. Thus, our data provide support for the idea that carefully controlled alterations in calorie intake and/or composition, designed to attenuate catch-up growth and accretion of adipose tissue, may be clinically beneficial in LBW individuals, in order to minimise the risk of adult disease. Future investigations will be critical in identifying mechanisms and markers of postnatal catch-up fat accretion, and in designing optimal nutritional interventions in key therapeutic windows, in order to prevent overnutrition in high-risk infants.

Acknowledgements We gratefully acknowledge grant support to $\mathrm{M}$. E. Patti from NIDDK (DK 062948), the American Diabetes Association and the Adler Foundation, and the resources from the Joslin Diabetes and Education Research Center (P30 DK36836).

\section{References}

1. Hales CN, Barker DJ, Clark PM et al (1991) Fetal and infant growth and impaired glucose tolerance at age 64. BMJ 303:1019-1022

2. Hofman PL, Cutfield WS, Robinson EM et al (1997) Insulin resistance in short children with intrauterine growth retardation. J Clin Endocrinol Metab 82:402-406

3. McCance DR, Pettitt DJ, Hanson RL, Jacobsson LT, Knowler WC, Bennett PH (1994) Birth weight and non-insulin dependent diabetes: thrifty genotype, thrifty phenotype, or surviving small baby genotype? BMJ 308:942-945

4. Hales CN, Barker DJ (1992) Type 2 (non-insulin-dependent) diabetes mellitus: the thrifty phenotype hypothesis. Diabetologia 35:595-601

5. Ong KK, Petry CJ, Emmett PM et al (2004) Insulin sensitivity and secretion in normal children related to size at birth, postnatal growth, and plasma insulin-like growth factor-I levels. Diabetologia 47:1064-1070

6. Cameron N, Demerath EW (2002) Critical periods in human growth and their relationship to diseases of aging. Am J Phys Anthropol Suppl 35:159-184

7. Dulloo AG (2005) A role for suppressed skeletal muscle thermogenesis in pathways from weight fluctuations to the insulin resistance syndrome. Acta Physiol Scand 184:295-307

8. Huxley RR, Shiell AW, Law CM (2000) The role of the size at birth and post natal catch-up growth in determining systolic blood pressure: a systematic review of the literature. J Hypertens $18: 815-831$

9. Crescenzo R, Samec S, Antic V et al (2003) A role for suppressed thermogenesis favoring catch-up fat in the pathophysiology of catch-up growth. Diabetes 52:1090-1097

10. Gafni RI, Baron J (2000) Catch-up growth: possible mechanisms. Pediatr Nephrol 14:616-619
11. Eriksson JG, Forsen T, Tuomilehto J, Winter PD, Osmond C, Barker DJ (1999) Catch-up growth in childhood and death from coronary heart disease: longitudinal study. BMJ 318:427-431

12. Cianfarani S, Germani D, Branca F (1999) Low birthweight and adult insulin resistance: the 'catch-up growth' hypothesis. Arch Dis Child Fetal Neonatal Ed 81:F71-F73

13. Ong KK, Ahmed ML, Emmett PM, Preece MA, Dunger DB (2000) Association between postnatal catch-up growth and obesity in childhood: prospective cohort study. BMJ 320:967-971

14. Singhal A, Cole TJ, Fewtrell M, Deanfield J, Lucas A (2004) Is slower early growth beneficial for long-term cardiovascular health? Circulation 109:1108-1113

15. Jimenez-Chillaron JC, Hernandez-Valencia M, Reamer $\mathrm{C}$ et al (2005) Beta-cell secretory dysfunction in the pathogenesis of low birth weight-associated diabetes: a murine model. Diabetes $54: 702-711$

16. Nagy TR, Clair AL (2000) Precision and accuracy of dual-energy $\mathrm{X}$-ray absorptiometry for determining in vivo body composition of mice. Obes Res 8:392-398

17. Gluckman PD, Hanson MA (2004) Living with the past: evolution, development, and patterns of disease. Science 305:1733-1736

18. Petry CJ, Ozanne SE, Wang CL, Hales CN (1997) Early protein restriction and obesity independently induce hypertension in 1year-old rats. Clin Sci (Lond) 93:147-152

19. Ozanne SE, Hales CN (2004) Lifespan: catch-up growth and obesity in male mice. Nature $427: 411-412$

20. Ozanne SE, Nicholas HC (2005) Poor fetal growth followed by rapid postnatal catch-up growth leads to premature death. Mech Ageing Dev 126:852-854

21. Wyrwoll CS, Mark PJ, Mori TA, Puddey IB, Waddell BJ (2006) Prevention of programmed hyperleptinemia and hypertension by postnatal dietary \{omega\}-3 fatty acids. Endocrinology 147: 599-606

22. Hahn P (1984) Effect of litter size on plasma cholesterol and insulin and some liver and adipose tissue enzymes in adult rodents. J Nutr 114:1231-1234

23. Yura S, Itoh H, Sagawa N et al (2005) Role of premature leptin surge in obesity resulting from intrauterine undernutrition. Cell Metab 1:371-378

24. Vickers MH, Gluckman PD, Coveny AH et al (2005) Neonatal leptin treatment reverses developmental programming. Endocrinology 146:4211-4216

25. Myers MG Jr, Patti ME, Leshan RL (2005) Hitting the target: leptin and perinatal nutrition in the predisposition to obesity. Endocrinology 146:4209-4210

26. Singhal A, Fewtrell M, Cole TJ, Lucas A (2003) Low nutrient intake and early growth for later insulin resistance in adolescents born preterm. Lancet 361:1089-1097

27. Axelsson IE, Ivarsson SA, Raiha NC (1989) Protein intake in early infancy: effects on plasma amino acid concentrations, insulin metabolism, and growth. Pediatr Res 26:614-617

28. Martin RM, McCarthy A, Smith GD, Davies DP, Ben Shlomo Y (2003) Infant nutrition and blood pressure in early adulthood: the Barry Caerphilly Growth Study. Am J Clin Nutr 77:1489-1497

29. Guesry P (1998) The role of nutrition in brain development. Prev Med 27:189-194

30. Fernstrom JD (2000) Can nutrient supplements modify brain function? Am J Clin Nutr 71:1669s-1675s

31. De Silva A, Jones PW, Spencer SA (2004) Does human milk reduce infection rates in preterm infants? A systematic review. Arch Dis Child Fetal Neonatal Ed 89:F509-F513

32. Ong KK, Dunger DB (2004) Birth weight, infant growth and insulin resistance. Eur J Endocrinol 151(Suppl 3):U131-U139

33. Stettler N, Stallings VA, Troxel AB et al (2005) Weight gain in the first week of life and overweight in adulthood: a cohort study of 
European American subjects fed infant formula. Cirulation 111:1897-1903

34. Soto N, Bazaes RA, Pena V et al (2003) Insulin sensitivity and secretion are related to catch-up growth in small-for-gestationalage infants at age 1 year: results from a prospective cohort. J Clin Endocrinol Metab 88:3645-3650

35. Mericq V, Ong KK, Bazaes R et al (2005) Longitudinal changes in insulin sensitivity and secretion from birth to age three years in small- and appropriate-for-gestational-age children. Diabetologia 48:2609-2614

36. Ezzahir N, Alberti C, Deghmoun S et al (2005) Time course of catch-up in adiposity influences adult anthropometry in individuals who were born small for gestational age. Pediatr Res 58:243-247

37. Eriksson JG, Forsen T, Tuomilehto J, Osmond C, Barker DJ (2001) Early growth and coronary heart disease in later life: longitudinal study. BMJ 322:949-953
38. Bhargava SK, Sachdev HS, Fall CH et al (2004) Relation of serial changes in childhood body-mass index to impaired glucose tolerance in young adulthood. N Engl J Med 350:865-875

39. Stettler N, Kumanyika SK, Katz SH, Zemel BS, Stallings VA (2003) Rapid weight gain during infancy and obesity in young adulthood in a cohort of African Americans. Am J Clin Nutr 77:1374-1378

40. Cettour-Rose P, Samec S, Russell AP et al (2005) Redistribution of glucose from skeletal muscle to adipose tissue during catch-up fat: a link between catch-up growth and later metabolic syndrome. Diabetes 54:751-756

41. Tronche F, Opherk C, Moriggl R et al (2004) Glucocorticoid receptor function in hepatocytes is essential to promote postnatal body growth. Genes Dev 18:492-497

42. Baron J, Klein KO, Colli MJ et al (1994) Catch-up growth after glucocorticoid excess: a mechanism intrinsic to the growth plate. Endocrinology 135:1367-1371 